\title{
Correction to: Patient perceptions of acute pain and activity disruption following inguinal hernia repair: a propensity-matched comparison of robotic-assisted, laparoscopic, and open approaches
}

\author{
James G. Bittner IV ${ }^{1} \cdot$ Lawrence W. Cesnik $^{2} \cdot$ Thomas Kirwan $^{3} \cdot$ Laurie Wolf $^{3} \cdot$ Dongjing Guo $^{4}$
}

Published online: 18 June 2018

(c) The Author(s) 2018

\section{Correction to: Journal of Robotic Surgery https://doi.org/10.1007/s11701-018-0790-9}

Unfortunately, the online published article has errors in Table 2. The number "118.0" found under the column
"L-IHR" and row "Time from IHR to resume activities" should be corrected to 18.0 .

The correct Table 2 is given below.

Table 2 Time variables related to postoperative groin pain and activity level (propensity matched)

\begin{tabular}{|c|c|c|c|c|c|c|c|c|c|c|}
\hline \multirow[t]{3}{*}{ Measures } & \multicolumn{5}{|c|}{ Robotic vs laparoscopic } & \multicolumn{5}{|c|}{ Robotic vs open } \\
\hline & \multicolumn{2}{|c|}{ R-IHR $(n=83)$} & \multicolumn{2}{|c|}{ L-IHR $(n=83)$} & \multirow[t]{2}{*}{$p$ value } & \multicolumn{2}{|c|}{ R-IHR $(n=85)$} & \multicolumn{2}{|c|}{ O-IHR $(n=85)$} & \multirow[t]{2}{*}{$p$ value } \\
\hline & $n$ & Mean (SE) & $n$ & Mean (SE) & & $n$ & Mean (SE) & $n$ & Mean (SE) & \\
\hline Time from IHR to survey (months) & 83 & $5.7(0.3)$ & 83 & $6.0(0.3)$ & 0.30 & 85 & $5.7(0.3)$ & 85 & $6.7(0.3)$ & 0.03 \\
\hline \multicolumn{11}{|l|}{ Pain } \\
\hline Time from IHR to little or no pain (days) & 74 & $15.5(1.6)$ & 79 & $14.0(1.4)$ & 0.17 & 76 & $15.5(1.5)$ & 75 & $18.2(2.0)$ & 0.33 \\
\hline Time from IHR to no Rx pain medications (days) & 66 & $9.4(1.5)$ & 66 & $11.6(1.7)$ & 0.30 & 69 & $9.4(1.4)$ & 69 & $10.6(1.2)$ & 0.03 \\
\hline \multicolumn{11}{|l|}{ Activity level } \\
\hline Time from IHR to resume activities (days) & 79 & $18.0(1.6)$ & 79 & $18.0(1.8)$ & 0.60 & 81 & $18.0(1.5)$ & 78 & $20.2(1.6)$ & 0.34 \\
\hline Time from IHR to return to work (days) & 60 & $17.8(2.1)$ & 54 & $17.9(2.8)$ & 0.34 & 64 & $17.0(2.0)$ & 44 & $21.7(2.4)$ & 0.08 \\
\hline Number of follow-up visits after IHR & 83 & $1.6(0.1)$ & 83 & $1.5(0.1)$ & 0.51 & 85 & $1.6(0.1)$ & 85 & $1.8(0.1)$ & 0.08 \\
\hline
\end{tabular}

$n$ number of respondents, $I H R$ inguinal hernia repair, $L$ laparoscopic, $O$ open, $R$ robotic assisted, $R x$ prescription, $S E$ standard error

The $p$ values were calculated using Wilcoxon rank sum test

The original article can be found online at https://doi.org/10.1007/ s11701-018-0790-9.

James G. Bittner IV

jbittner4@gmail.com

1 Department of Surgery, Virginia Commonwealth University School of Medicine, PO Box 980519, Richmond, VA 23298, USA

2 Market Research Department, Intuitive Surgical, Inc., Sunnyvale, CA, USA

3 Bruno and Ridgway Research Associates, Lawrenceville, NJ, USA

4 Department of Clinical Affairs, Intuitive Surgical, Inc., Sunnyvale, CA, USA
Open Access This article is distributed under the terms of the Creative Commons Attribution 4.0 International License (http://creativeco mmons.org/licenses/by/4.0/), which permits unrestricted use, distribution, and reproduction in any medium, provided you give appropriate credit to the original author(s) and the source, provide a link to the Creative Commons license, and indicate if changes were made. 